\title{
Democratización de la información en Chile. El lugar de la apropiación social de las TIC en la agenda digital 2004-2006"
}

\section{Valeria CABRERA ${ }^{* *}$}

La "democracia interactiva" es, hoy, los proyectos de "ciudades cableadas" (wired city) que los generadores de ideas (thinks tanks) proponen y que se convierten en el lugar de experimentación de la ideología tecnocomunitarista.

Armand Mattelart, en El mito Internet, 2002.

La política pública de TIC en Chile, contenida fundamentalmente en la Agenda Digital 2004-2006 ${ }^{1}$, AD, se enmarca dentro de los acuerdos de la Primera Cumbre Mundial de la Sociedad de la Información realizada en Ginebra el año $2003^{2}$. En esta última, se consideró que uno de los mayores problemas existentes después de la explosión de las TIC y más específicamente de Internet a nivel mundial, es la brecha digital que se da entre países y dentro de éstos. Para acortar la brecha digital, esto es, para reducir la diferencia de oportunidades entre quienes tienen acceso a las TIC y quienes no, la $\mathrm{AD}$ ha comprometido grandes esfuerzos y recursos en la generación de programas y proyectos orientados a la masificación del acceso y a la alfabetización digital del mayor número posible de ciudadanos.

Asimismo, el desarrollo de las TIC se ha convertido en un factor de central importancia dentro del proceso de Modernización del Estado chileno. Desde el año 2000, el gobierno se ha ocupado de expandir la implementación de las TIC a lo largo del territorio nacional de acuerdo a dos motivaciones fundamentales: por un lado, porque el actual sistema económico globalizado ha exigido que el país acelere su competitividad tecnológica comercial y productiva; y por otro, porque el proceso de modernización consiste en descentralizar los poderes del Estado y democratizar el acceso a los bienes y servicios públicos.

Estos lineamientos han implicado a las TIC dentro de un proyecto-país que no puede desconocer los problemas sociales que están involucrados en él. De acuerdo a ello, la implementación de puntos de acceso universal y la alfabetización digital ocupan una parte importantísima dentro de la AD, planeándose como las iniciativas tecnológicas de corte social que buscan garantizar el derecho a la comunicación y a la información. Con esto, lo que se pretende es que la condición social, económica y cultural de las personas no

\footnotetext{
* Este artículo es resultado de la investigación de tesis “Apropiación Social en la Implementación Comunitaria de TIC. Identidad, desarrollo y participación ciudadana en la experiencia de la Red de Información Comunitaria de la Araucanía", financiada por el Centro de Investigaciones para el Desarrollo, CIIE /IDRC, Ottawa, Canadá.

*** Licenciada en Filosofía y Magíster en Comunicación y Sociedad con mención en Políticas Públicas para Internet, Flacso-Ecuador

1 GAD. Agenda Digital Chile. 2003. En: http://www.agendadigital.cl.

${ }^{2}$ La Segunda Cumbre Mundial de la Sociedad de la Información se celebró el 2005 en Túnez
} 
sea un impedimento para acceder a las TIC y a Internet quedando, de este modo, el derecho a la información y a la comunicación debidamente garantizado (de manera exponencialmente equitativa en la medida en que los puntos de acceso universal y la alfabetización digital aumenten). Y por otro lado, de acuerdo a La Declaración Milenio de la ONU en el año 2000, al Informe sobre Desarrollo Humano 2001 del PNUD $^{3}$ y los acuerdos de la Cumbre de Ginebra 2003, la AD considera que las TIC son una herramienta para el desarrollo humano, por lo que con el acceso a ellas se abre a los usuarios la posibilidad de utilizarlas para contribuir al enriquecimiento de su vida individual y comunitaria.

En tales sentidos, la Agenda Digital 2004-2006, tiene como objetivo “...contribuir al desarrollo de Chile mediante el empleo de las tecnologías de información y comunicación (TIC) para incrementar la competitividad, la igualdad de oportunidades, las libertades individuales, la calidad de vida y la eficiencia y transparencia del sector público, enriqueciendo al mismo tiempo la identidad cultural de la nación y de los pueblos originarios" $"$.

La AD cuenta con un Plan de Acción dividido en siete lineamientos estratégicos con treinta y cuatro iniciativas. Siendo sus líneas estratégicas de dos tipos. Las primeras son de alto impacto en corto plazo, entre las que se cuentan: acceso universal; educación y capacitación; gobierno electrónico. Las segundas son de importancia estratégica pero de maduración más lenta: despegue de la industria TIC; marco jurídico-normativo; seguridad de la infraestructura de la información.

\section{Incorporación a la Sociedad Red}

Para efectos del presente análisis, nos detendremos brevemente la línea estratégica de alto impacto en corto plazo: de acuerdo con el plan de acceso universal, se estableció la meta de consolidar la Red Nacional de Infocentros y cyber-cafés asegurando que los 1.300 infocentros hoy operativos tengan banda ancha al terminar el 2006; además, se pretende conseguir que 900 mil hogares tengan conexión a Internet, esto implica acuerdos con el sector privado y la generación de un marco jurídico adecuado para reducir los costos de conectividad principalmente orientados a hogares de ingresos medios del país. En relación a la educación y capacitación digital, el objetivo es lograr que un millón de personas sean alfabetizadas digitalmente para el uso de Internet y la realización de trámites en los servicios públicos on line, recibiendo a su vez una certificación que acredite su competencia digital. Para lograr este objetivo se cuenta con la cooperación conjunta de universidades, organizaciones técnicas de capacitación, la Red Enlaces y el programa de Alfabetización Digital. En tercer término, en lo relativo al gobierno electrónico, el objetivo es masificar los trámites electrónicos, extendiendo las TIC a todos los poderes del Estado, la meta es llegar a tener 300 trámites en línea de consulta ciudadana en Internet y avanzar hacia una fase más interactiva del Gobierno Electrónico.

Es sobre esta concepción, que considera las TIC una herramienta central para el avance social, que los programas de acceso universal del país proliferaron rápidamente.

\footnotetext{
${ }^{3}$ Esto, de acuerdo al Informe sobre el desarrollo humano 2001. Poner el adelanto tecnológico al servicio del desarrollo humano. Pág. 1-9. PNUD. En: http://www.undp.org/hdr2001/spanish/.

${ }^{4}$ GAD. Agenda Digital Chile 2003. http://www.agendadigital.cl.
} 
Los índices de conectividad, la cantidad de puntos de acceso comunitario a las TIC $^{5}$ y el número de alfabetizados digitales se mantienen en un aumento sostenido ${ }^{6}$. Se avanzó y se sigue avanzando hacia los sectores rurales, se alfabetizan digitalmente comunidades indígenas, personas con discapacidad física, dueñas de casa, personas de la tercera edad, artesanos, comerciantes, desocupados, jóvenes y niños bajo el ideal de la democratización de la información. Asimismo, más actores sociales se comprometen en el recorrido que avanza hacia dicha tecnologización: universidades, organismos internacionales, el sector privado, fundaciones, gobiernos regionales y municipales han aportado recursos de gestión, técnicos y materiales para levantar puntos de acceso universal y garantizar a la ciudadanía, sin distinciones de tipo alguno, su incorporación a la sociedad red.

Los beneficios que se esperan de la implementación de las TIC parecen estar relativamente claros, sin embargo, los proyectos puestos en marcha no logran aún, después de un par de años de funcionamiento, conseguir un impacto profundo en las realidades de las personas que utilizan los puntos de acceso universal y se han alfabetizado digitalmente. Los logros de la "agenda corta" de la $\mathrm{AD}$, nos proporcionan ya la información necesaria para elaborar interrogantes fundamentales acerca del impacto que las tecnologías tienen efectivamente en la superación de las realidades más pobres de nuestras sociedades, tal como se plantea desde el ideal de la democratización de la información y el cierre de la brecha digital. Hasta el momento, persiste la ausencia de antecedentes sobre cuál es la relación entre la política pública de TIC y su impacto social real.

¿La ampliación del acceso gratuito y la alfabetización digital garantizan el cierre de las brechas sociales como las de género, edad, pertenencia étnica y lugar de origen? ¿Cómo lograr una apropiación social de las TIC en personas con escaso capital cultural? ¿Cómo generar flujos de información relevante socialmente? ¿Qué actores sociales se comprometen a generar un soporte para la inclusión social y no sólo digital de estas personas alfabetizadas digitalmente? La impresión que queda es que todavía no sabemos mucho acerca del impacto social que el uso de las TIC está reportando a sus usuarios.

Quienes respaldan la implementación de TIC con sentido social, parten de la base que asegura que, particularmente los recursos de Internet, pueden producir cambios concretos en el entorno de los usuarios en términos de desarrollo, participación y cultura y sostienen que para que esto sea posible, es necesaria la apropiación social de las TIC Esto implicaría que los usuarios utilicen las TIC de tal modo que sus prácticas logren intervenir positivamente en sus propias realidades.

\section{Apropiación Tecnológica}

Martín-Barbero define la "apropiación" como el fenómeno caracterizado por la adecuación que los individuos hacen de determinados productos culturales a su propia forma de percibir el mundo y de intervenirlo ${ }^{7}$. Asimismo, se habla de apropiación tecnológica cuando los individuos comprenden los códigos y significados de las TIC

\footnotetext{
${ }^{5}$ Catalogado por la UIT como "medio alto". En: UIT. Informe sobre el Desarrollo Mundial de las Telecomunicaciones. Indicadores de acceso para la sociedad de la información. Resumen de conclusiones. Pág. 22. 2003. http://www.itu.int/ITU-D/ict/publications/wtdr_03/material/WTDR03Sum_s.pdf.

${ }^{6}$ La meta de la AD para el año 2006 era de 500 mil alfabetizados digitales, esta cifra fue superada en el 2005 con un total de 727 mil personas "infoalfabetizadas" más 36 mil nuevos infoalfabetizados a mayo de 2006. Ibid.

${ }^{7}$ MARTÍN-BARBERO, Jesús. Dinámicas urbanas de la cultura. En: Revista Gaceta de Colcultura No 12. Instituto Colombiano de Cultura. 1991.
} 
siendo capaces de utilizarlas de acuerdo a sus propios intereses y necesidades ${ }^{8}$. En este sentido, cabe hacer una diferenciación entre apropiación y «uso», entendiendo por este último el fenómeno según el cual los individuos sincronizan con las TIC sin mediar un proceso previo de reconocimiento de sí mismos y de lo otro que les permita interiorizar plenamente sus significaciones y sus utilidades. Si se considera que el acceso a las TIC y la alfabetización social son una plataforma tecnológica que sustenta un proceso de comunicación que, en cuanto tal es absolutamente complejo ¿quiénes están analizando este proceso? ¿Qué indicadores utiliza la AD para medir los niveles de apropiación tecnológica de la gran masa de alfabetizados digitales del país?

$\mathrm{Si}$, de acuerdo a Martín Barbero, la apropiación se genera en la medida en que se produce una adecuación entre individuos y productos culturales, como es el caso de los flujos de información en Internet, se hace necesario analizar el acceso y uso de las TIC desde un punto de vista que vaya más allá de lo meramente tecnológico e instrumental, en este caso, desde un punto de vista comunicacional y social. Hall ${ }^{9}$ sostiene que el recorrido de un discurso en el proceso comunicativo, sólo se completa cuando redunda en prácticas sociales, y que este recorrido depende fundamentalmente de la traducción de los códigos y de sus significaciones. Para que esto sea posible, el individuo que decodifica debe conocer los significados del discurso, de lo contrario, no puede seguir la lógica del mismo y por lo tanto, éste no tiene efecto. En este sentido, la importancia de avanzar en el conocimiento y planeación de los flujos de información se hace sumamente relevante al analizar el acceso y uso de las TIC y las posibilidades de su apropiación social.

Actualmente las evaluaciones en materia de TIC en Chile tienen una orientación predominantemente cuantitativa. Esta tendencia generalizada -que considera que la brecha digital aumenta o disminuye en relación directa con el número de PCs por hogares, el número de éstos que tienen conexión a Internet, la cantidad de puntos de acceso universal y el número de alfabetizados digitales-, ha impedido que los índices de conectividad y de masificación del acceso incluyan información desagregada y relevante sobre el impacto que la implementación de TIC tiene en lo relativo a los manejos sociales que logran sus usuarios. Además, esta misma orientación no ha logrado incluir de manera coherente el discurso que sustenta la necesidad de la inclusión digital, según el cual, se considera que las TIC, al ser un instrumento de comunicación social mediante el cual se producen relaciones sociales en la interacción entre personas iguales y diversas culturalmente, en la interacción entre comunidades locales y el resto del mundo y entre la ciudadanía y el Estado, exigen también la atención, observación y análisis de los efectos que su implementación y uso generan en la cotidianidad individual y social de sus usuarios.

Con todo, el enfoque social de la implementación de TIC se ha patentizado en un gran número de experiencias en el país, sin embargo, las condiciones que garanticen un impacto real y positivo en términos de apropiación social siguen siendo motivo de incertidumbre. Aunque las políticas de masificación del acceso y alfabetización digital aportan al cierre de la brecha digital en tanto propician el derecho a la comunicación como plataforma para la democratización de la información, todavía no se ve hasta qué punto

\footnotetext{
${ }^{8}$ GARRIDO et al. Red de Información Comunitaria: Siete años aportando al cierre de la brecha digital en la Araucanía. Pág. 93. Flacso Chile. Santiago. Primera Edición, 2005.

${ }^{9}$ HALL, Stuart. Codificar y Decodificar. En: Cultura, Media y Lenguaje. Pág. 129-139. London, Hutchinson. 1980. http//www.nombrefalso.com.ar/materias/apuntespdf/hall-3.pdf.
} 
esta equidad y este derecho redunden en nuevas prácticas sociales en quienes han sido alfabetizados.

Pese a que la preocupación por el impacto social de las TIC está creciendo, todavía no se ha generado una línea de implementación y de análisis definidamente cualitativo. Si bien, varias investigaciones existentes en esta materia mantienen una preocupación constante en torno a determinado número de factores problemáticos que surgen de la relación entre masificación de las TIC y su apropiación social, sigue predominando el modo cuantitativo de asumirla tanto desde la política pública y como problema de análisis social.

\section{Conclusión}

De acuerdo a lo planteado, sólo se puede sostener que la política nacional de TIC ha empeñado gran parte de sus esfuerzos en conseguir dos objetivos simultáneos: la ampliación de la conectividad y la masificación del acceso. En el primer caso, se trata de una inversión en recursos tecnológicos que implican el incremento de circuitos de conexión a Internet en la mayor parte del territorio chileno y la adquisición de diversos hardware y software que garanticen las condiciones materiales para el acceso a la información en red. En segundo término, dadas las condiciones de conectividad, la masificación del acceso consiste en hacer efectivo el acercamiento de la población al uso de las TIC mediante la alfabetización digital. El punto crítico de la política pública de TIC y de la AD chilena siguen siendo las condiciones de posibilidad que éstas generan para producir una apropiación tecnológica en los sectores de la población acceden a las TIC por medio de los programas de masificación. Aunque las cifras nacionales e internacionales que determinan la brecha digital se sostienen básicamente en la relación numérica entre quienes acceden y quienes no acceden a las TIC, las ventajas que éstas mismas traigan a la población apuntan fundamentalmente a los niveles de apropiación social.

La apropiación social de las TIC, por lo tanto, es un tema pendiente en la política pública de TIC en Chile. La Agenda Digital 2004-2006 no estableció especificaciones teóricas ni prácticas acerca de las distintas orientaciones en que pueden ser entendidas la brecha digital y la democratización información. Por el contrario, en ella se tiende a identificar la brecha digital con un fenómeno cuya solución se encuentra en el aumento de puntos de acceso, de alfabetización digital y de la información pública en línea. La AD, responde por tanto, a una disposición predominantemente instrumental de implementación de TIC, que pese a que las considera una herramienta estratégica para el desarrollo humano, no incorpora mecanismos de traspaso simbólico ni cultural que garanticen su apropiación social.

Siguiendo los análisis hechos por Martín-Barbero, y de acuerdo con lo que plantea también Armand Mattelart ${ }^{10}$, frente a todo este nuevo ambiente de reestructuraciones y transformaciones, también es totalmente necesario considerar que "las tecnologías no son neutras". Por el contrario, en este nuevo espacio llamado sociedad red, las TIC se convierten en el principal enclave de concentración de flujos de intereses económicos y políticos que atraviesan a su vez las esferas sociales, los conflictos culturales y sus

\footnotetext{
${ }^{10}$ MATTELART, Armand. Cómo nació el mito Internet. En: El Mito Internet. Pág. 25-32. Editorial Aún Creemos en los Sueños. Santiago de Chile. Primera Edición, 2002.
} 
procesos de comunicación ${ }^{11}$. ¿Cuál es, entonces, el valor social de la democratización de la información? Asumir que las TIC sean una herramienta para el desarrollo, no implica, por lo tanto, desconocer que tras el fenómeno de la brecha digital siguen operando mecanismos de reproducción del orden dominante, en donde las relaciones de poder entre países ricos y pobres, entre Estado y ciudadanía, entre instituciones e individuos son desiguales y la circulación de contenidos se da de manera asimétrica ${ }^{12}$. Todo parece indicar que lo que falta es una valoración estratégica de la información socialmente relevante. Para lo cual es necesario realizar un giro que ubique el valor de la información y no el de las tecnologías, a la base de la producción, circulación y manejo de los contenidos significativos o socialmente apropiables que permitan, a su vez, la construcción de espacios reales para consolidar redes de información y comunicación.

\section{Referencias bibliográficas}

BONILLA, M. y CLICHE, G. Investigación para sustentar el diálogo sobre el impacto de Internet en la sociedad latinoamericana y caribeña. En: BONILLA M. y G. CLICHE editores. Internet y Sociedad en América Latina y el Caribe. Flacso Sede Ecuador- IDRC. Primera Edición, 2001.

GARRIDO et al. Red de Información Comunitaria: Siete años aportando al cierre de la brecha digital en la Araucanía. Flacso Chile. Santiago. Primera Edición, 2005.

GRUPO DE ACCIÓN DIGITAL, GAD. Agenda Digital Chile. 2003. En: http://www.agendadigital.cl.

HALL, Stuart. Codificar y Decodificar. En: Cultura, Media y Lenguaje. London, Hutchinson. 1980. http://www.nombrefalso.com.ar/materias/apuntespdf/hall-3.pdf.

MARTÍN-BARBERO, Jesús. Dinámicas urbanas de la cultura. En: Revista Gaceta de Colcultura $\mathrm{N}^{\circ}$ 12. Instituto Colombiano de Cultura. 1991.

MARTÍN-BARBERO, Jesús. Tecnicidades, identidades, alteridades: des-ubicaciones y opacidades de la comunicación en el nuevo siglo. En: Diálogos de la Comunicación $\mathrm{N}^{\mathrm{o}} 64$. Felafacs. Lima. 2002.

MATTELART, Armand. Cómo nació el mito Internet. En: El mito Internet. Editorial Aún Creemos en los Sueños. Santiago de Chile. Primera Edición, 2002.

PROGRAMA DE LAS NACIONES UNIDAS PARA EL DESARROLLO, PNUD. Informe sobre el desarrollo humano 2001. Poner el adelanto tecnológico al servicio del desarrollo humano. http://www.undp.org/hdr2001/spanish/.

UNIÓN INTERNACIONAL DE TELECOMUNICACIONES, UIT. Informe sobre el Desarrollo Mundial de las Telecomunicaciones. Indicadores de acceso para la sociedad de la información. Resumen. 2003. En:

http://www.itu.int/ITUD/ict/publications/wtdr_03/material/WTDR03Sum_s.pdf.

\footnotetext{
${ }^{11}$ MARTÍN-BARBERO, Jesús. Tecnicidades, identidades, alteridades: des-ubicaciones y opacidades de la comunicación en el nuevo siglo. En: Diálogos de la Comunicación No 64. Pág. 19. Felafacs. Lima. 2002.

${ }^{12}$ BONILLA, M. y CLICHE, G. Investigación para sustentar el diálogo sobre el impacto de Internet en la sociedad latinoamericana y caribeña. En: BONILLA, M. y CLICHE, G. (editores). Internet y Sociedad en América Latina y el Caribe. Pág. 15-36. Flacso Sede Ecuador- IDRC. Primera Edición, 2001.
} 